\title{
Leprosy of the eye-a general outline
}

\author{
VAN C JOFFRION \& MARGARET E BRAND \\ National Hansen's Disease Center, Carville, Louisiana, USA
}

Summary Ocular complications from leprosy (Hansen's disease) seriously threaten a patient's quality of life.

The eye (the anterior segment structures) may be infiltrated and damaged by the mycobacteria directly; may suffer damage from inflammation (Type II reaction), and be damaged as a result of changes in the extraocular structures. These are features of the disease at or near the lepromatous end of the spectrum.

In addition to direct damage, the eye in about $15-20 \%$ of patients irrespective of their disease type, is subject to abnormal exposure due to nerve damage. This may be motor (C.7) causing lagophthalmos, or sensory (terminal branches of ophthalmic division of C.5) causing corneal hypaesthesia. Secondary infection by other pathogens complicates the damage initiated by dryness and trauma.

The patient most at risk is the one with active, long-standing lepromatous disease. $\mathrm{He} / \mathrm{she}$ will possibly have diminished pain sensitivity. Symptoms will be unreliable. Routine objective examination of such patients becomes mandatory. Fortunately, most of the significant pathology is visible with the help of relatively unsophisticated instruments. A useful examination may be done in 2 or 3 minutes once the routine has become established.

An outline of pathophysiology, diagnosis and management of the main features of ocular leprosy have been presented in this article. Its aim has been to help the physician or other health worker to assume responsibility for the primary eye care of their leprosy patients and thus reduce the possibility of blindness. Loss of sensation of the extremities is a serious handicap to any individual. Blindness superimposes an intolerable burden.

\section{Introduction}

Leprosy is a disease which causes disability by affecting hands, feet and eyes. The purpose of this paper is to give an overall perspective on the effects of leprosy on the eye in a logical outline form and to indicate in a broad outline form what can go wrong, how to recognize it and how to manage the problem. Further reading and consultations with ophthalmologists are highly recommended in order to get a working knowledge of leprosy of the eye. 
The eyes are involved in leprosy in four ways:

Abnormal exposure of the eyes secondary to involvement of the 5th and 7th cranial nerves.

2 Infiltration of the eyes and/or eyelids by the leprosy bacillus.

3 Inflammation of the eyes secondary to the infiltration by the leprosy bacillus.

4 Complications secondary to involvement of neighbouring structures: eyelids, lacrimal glands and the naso-lacrimal drainage system.

Note. Not all patients will have all of these problems. The patient most at risk is the long-standing lepromatous case.

\section{Exposure}

Exposure has dual etiology:

1 Sensory loss - 5th nerve damage.

2 Motor loss-7th nerve damage.

The patient with sensory loss can blink, but does not as often as necessary or with full potential due to the lack of pain. The patient with motor loss wants to blink but cannot adequately. There can be varying combinations and degrees of damage to the two nerves. Tuberculoid, borderline and lepromatous cases can all be affected.

\section{SENSORY LOSS (5TH NERVE DAMAGE) PATHOPHYSIOLOGY}

Corneal and conjunctival anaesthesia. (It is actually a hypaesthesia, but the word anaesthesia is commonly used.) Injuries, dryness of the cornea, infections and inflammations are ignored by the patient due to a decreased sensation. Total anaesthesia is rare. But even partial anaesthesia (hypaesthesia) can seriously affect the prognosis.

\section{Diagnosis}

1 Observe the patient for an abnormality of spontaneous blinking.

2 Evaluate corneal sensation with a wisp of cotton.

3 Symptoms are not proportionate to the signs (due to anaesthesia).

4 Check the inferior aspect of cornea for the signs of dryness. Confirm this with fluorescein staining.

Management-See below with 7th nerve.

MOTOR LOSS (7TH NERVE DAMAGE)—PATHOPHYSIOLOGY

The orbicularis oculi muscles are paralysed to varying degrees when the 
zygomatic branch of the 7th nerve is affected. Lagophthalmos, or the inability to close the eyes, results in:

1 Drying of the eyes; especially while sleeping.

2 Exposure of the eyes to more danger due to the loss of protective blinking when the eyes are threatened.

3 Increase in eye infections and irritation due to lack of normal eyelid and tear physiology.

\section{Diagnosis}

1 Instruct the patient to close the eyes first as in sleep and then with maximal effort. There is weakness of the orbicularis muscle and possible lagophthalmos. 2 In spite of severe symptoms of dryness (burning, irritation and tearing) the eye may look fairly normal.

3 Evaluate the cornea by fluorescein staining.

\section{Management (sensory and motor loss)}

1 Educate the patient as to the dangers of the condition. He should be taught self-examination by checking for redness of the eyes with a mirror and monitoring his own vision. A dry cornea may decrease visual acuity by $50 \%$ or more.

2 Periodic clinical examinations.

3 Blinking exercises to strengthen unparalysed muscle fibres.

4 Artificial tears, e.g. methylcellulose, polyvinyl alcohol or castor oil. The patient with sensory loss must use drops regularly even if symptom-free. The patient with sensation will want to use the drops to relieve discomfort.

5 Taping the eyes shut at night, if indicated. (Caution: be sure to learn the proper technique before doing this so as not to damage the cornea.)

6 Protective eyeshield, if indicated. This forms a moisture chamber and protects the eye from trauma as well. (Used X-ray film is a good source of material to make eye-shield cups.)

7 Tarsorrhaphy, if indicated. A simple procedure; especially indicated in anaesthetic eyes showing corneal damage. A temporary tarsorrhaphy effective for 2-3 weeks can be achieved by a simple horizontal mattress suture across the palpebral fissure.

8 Temporalis muscle tendon transfer. Requires a skilled surgeon and a motivated patient who will use it regularly. Patients with anaesthetic eyes will not use it properly.

\section{Direct infection of the eye by Mycobacterium leprae}

\section{GENER AL INFORMATION}

This affects patients at the lepromatous end of disease spectrum. It is a 
blood-borne infection. The lesions are confined to the anterior segment structures of the eye (cooler tissues), i.e. the cornea, conjunctiva, episclera, sclera, iris and ciliary body. The lesions, or infiltrates, usually become visible 4 or more years after the onset of the disease. In the early phases of the disease this lepromatous infiltration of the eye causes little, if any inflammation. Symptoms are absent and the eyes are white; however, patients must be warned that the eyes, along with the skin and other organs, are likely to become inflamed later. With effective treatment the disease should eventually arrest. But if a relapse occurs, the new infiltrates will provoke serious and destructive inflammation. The patients most at risk are those with a history of 20 or more years of lepromatous disease.

\section{CORNEA PATHOPHYSIOLOGY}

By corneal microscopy, early lesions are seen in the corneal nerves and subepithelial layer. The infiltrates start superficially and peripherally in the upper temporal quadrant. As the disease advances irto other quadrants and the pupillary area, new infiltrates may develop peripherally in the deeper layers of the cornea. The lesions start discretely, but later coalesce. At first they are avascular, but secondary pannus may follow.

\section{Symptoms}

None, unless the pupillary area is involved reducing visual acuity.

\section{Signs}

Haziness of the cornea mostly in the upper temporal quadrant. 'Pearls' may be seen resembling chalk dust.

\section{Diagnosis}

Penlight, magnifying loupe. A darkened room is an advantage. The patient looks down and the examiner retracts the upper eyelid and focuses on the upper temporal quadrant.

\section{Management}

Systemic anti-leprosy drugs. With the disease under control infiltrates slowly resolve. Residual scarring may persist but visual acuity should improve somewhat. If a vascular pannus has formed, prognosis for an improvement of vision is guarded. Warn the patient about future inflammation, how to recognize it and what to do. (See section on inflammation, below.) 
IRIS

The earliest lesions are not visible until the tiny iris lepromata or 'pearls' form. They resemble chalky particles on the iris surface. They are first seen close to the pupillary margin. Larger, hypopigmented nodules are very occasionally seen in highly active, long-standing disease.

\section{Symptoms}

None.

\section{Diagnosis}

Penlight, darkened room, and focal oblique illumination. Large 'pearls' can be seen with a magnifying loupe, small ones require greater magnification (corneal microscope).

\section{Management}

1 Systemic anti-leprosy drugs.

2 Patient education about inflammation, its recognition, significance and need for treatment. Reinforce the need for compliance in taking anti-leprosy drugs.

\section{CILIARY BODY}

The earliest infiltrates probably start here. If the disease is very active, macroscopic nodules may form and grow to involve adjoining structures-the root of the iris and the overlying sclera.

\section{SCLERA, EPISCLERA AND CONJUNCTIVA}

The nodules which originate in the ciliary body appear on the surface near the temporal limbus (corneo-scleral junction) which is a cool, exposed part of the eye. They are painless. They have firm to rubbery texture and are pink to yellow in colour. They may extend into the cornea. Large nodules can interfere with the normal function of the eyelids in spreading the tears over the corneal surface. Nodules suggest very active disease. Their appearance, after anti-leprosy treatment has been fully established, suggests either non-compliance, a noneffective drug or drug resistance.

\section{Management}

1 Systemic anti-leprosy drugs. 
2 Superficial excision of large nodules to restore normal eyelid function. (This surgery requires trained personnel.)

3 Patient education about the need for compliance in taking anti-leprosy medications as ordered, and a warning about possible inflammation.

\section{INFLAMMATION OF THE EYE INFILTRATED WITH LEPROSY BACILLI}

This is the ocular counterpart of erythema nodosum leprosum (ENL) of the skin, and like ENL, it is a recurring phenomenon. It occurs only in the lepromatous form of the disease. Remember that there is a mycobacterial infiltration of the eye without inflammation which was just reviewed. The time interval between infiltration and inflammation may be as little as a few months, but could be as much as several years after the initial infiltration of the eye. This may occur when the bacterial index is declining but the antigen level is apparently high. It may continue to occur after the disease is 'inactive' by current criteria. It must be understood by personnel who treat the eye, that the eye contains very reactive tissues and often requires much longer periods of time to subdue inflammation and infection than other structures in the body such as the skin.

The structures affected are the iris and ciliary body (iritis, iridocyclitis or 'anterior' uveitis), episclera (episcleritis) and sclera (scleritis).

Note. The cornea is not affected unless previously vascularized.

\section{Warning}

If the sensation of pain is impaired, symptoms are unreliable. Therefore, objective examinations are mandatory. The patient must practice monitoring of his own visual acuity. It may be his only index of trouble.

EPISCLERITIS

Diagnosis and symptoms

Moderate pain especially on eye movement.

\section{Signs}

Redness, usually in discrete patches, Dull red. Patches are very tender. (Palpate these patches through the eyelids.) Retract the eyelids to examine fully all quadrants of the eye.

\section{Management}

Steroid drops. Apply them hourly, initially, then reduce the frequency as fast as 
the condition permits. Steroid ointments are valuable for night-time use or when the condition is less acute. Warm-compresses are comforting and possibly therapeutic.

\section{SCLERITIS}

A serious complication which can lead to scleral perforation. It is less common than episcleritis. It may be associated with intraocular inflammation.

\section{Diagnosis and symptoms}

Severe deep pain. Distribution of the pain is circumorbital and radiating back to the temple.

\section{Signs}

Redness, with mauve to grey tinge. Usually in discrete patches. Distribution is varied but commonly over the ciliary body area. Affected areas are exquisitely tender. The diagnosis can be made by a penlight and gentle palpation through the eyelid.

\section{Complications}

Scleral thinning or actual perforation. Appear later as slate-grey areas deep to the conjunctiva.

\section{Management}

1 Topical steroid drops are helpful, but inadequate if used alone.

2 Subconjunctival steroid injections.

Note: Do not inject in the immediate area of the inflammation. Use the soluble form of the drug, not the depot form. Personnel must be trained in the technique. 3 Systemic steroids and/or other systemic anti-inflammatory agents.

4 Analgesics and warm compresses for pain as required.

IRIDOCYLITIS (IRITIS, 'ANTERIOR' UVEITIS)

\section{The principle single cause of blindness in leprosy}

Intermittent attacks may occur over many years. Between the acute attacks or exacerbations, low-grade inflammation without symptoms may persist causing adhesions and later complications, e.g. secondary glaucoma. Other complica- 
tions include cataract, iris and ciliary body atrophy and, finally, phthisis bulbi (soft, blind eye).

\section{Diagnosis and symptoms}

Decreased vision, pain of the whole eye, photophobia and tearing.

\section{Signs}

Eyelid swelling, red eye, tender eye, ciliary flush (circum-corneal injection), corneal oedema (steamy), cloudy aqueous humour, keratic precipitates (deposits on the internal surface of the cornea), poorly reactive small pupil, posterior synechiae (adhesions of iris to lens), irregular pupil (especially on attempted dilation with mydriatics), iris atrophy (advanced cases), eccentric pupil (advanced cases). Increased intraocular tension in some cases.

Note: The entire anterior segment of the eye is involved to varying degrees. Signs written in italics are found frequently and are most easily recognized by the practitioner without the benefit of formal ophthalmic training or sophisticated equipment.

\section{Management}

1 Effective anti-leprosy treatment.

2 Atropine eye drops or ointment twice a day.

3 Steroid eye drops or ointment. Use drops hourly, initially. Reduce frequency as fast as continuing control permits. Use ointment two to four times a day.

Note: Drops are generally more effective, easier to explain to the patient, and more economical as there is less waste. If the patient has trachoma or corneal disease also, use an appropriate topical antibiotic.

4 Systemic steroids if the uveitis is severe. Start with prednisone $60 \mathrm{mg}$ per day and taper the dosage over approximately 2 weeks in most cases. Use for longer if indicated. (Thalidomide is a useful adjunct provided proper precautions are taken for female patients of child-bearing age.)

5 Subconjunctival steroid injection if the uveitis is severe. (One needs instruction to administer this injection.)

6 If the intraocular tension is elevated, use acetazolamide (Diamox) $250 \mathrm{mg}$ four times a day. (Do not use pilocarpine. It counteracts atropine.)

7 Intraocular tension needs to be monitored at least bi-weekly while uveitis is active, or while steroids are being used topically. (Steroid-induced glaucoma can occur.)

EXTRA-OCULAR INVOLVEMENT: MADAROSIS

Pathophysiology. Destruction of hair follicles by leprosy bacilli. 


\section{Diagnosis}

Loss of eyebrows and/or eyelashes.

\section{Management}

1 Systemic leprosy treatment.

2 Cosmetic eyebrow transplants from the scalp.

Note. The hair loss is permanent; however, a hair transplant will take if done properly. A neuro-vascular island pedicle flap is one of the best procedures.

\section{DACRYOCYSTITIS-PATHOPHYSIOLOGY}

Obstruction of the naso-lacrimal duct secondary to destruction of nasal mucosa and nasal bone absorption. May be acute, progressing to abscess formation, rupture and fistula formation. More commonly it presents as low-grade infection, symptomless except for some swelling and a chronic watery, muco-purulent discharge in the conjunctival sac. This chronic source of infection, often overlooked, is a potential danger to the cornea already compromised by exposure and the increased risk of trauma. Corneal ulceration is then a probability. It also increases the risk of postoperative ocular infection in eyes subjected to surgery. Endophthalmitis may ensue in intraocular surgical cases.

\section{Diagnosis, symptoms and signs}

\section{Acute infection}

Pain, swelling, tenderness (maximal in the lacrimal sac area), lacrimation with or without purulent exudate.

\section{Chronic infection}

Lacrimation with purulent exudate increased by pressure over the lacrimal area. Slight swelling and tenderness. Obstruction to irrigation of the naso-lacrimal system.

\section{Management}

For the acute condition:

1 Hot compresses.

2 Systemic antibiotics.

For the chronic condition:

1 Gentle digital expression of the pus through the puncta. If it does not come readily do not force it. The canaliculi (between the puncta and lacrimal sac) may also be obstructed.

2 Daily irrigation of lacrimal sac using saline then antibiotic ophthalmic solution, for 2 weeks. 
3 Dacryocystorrhinostomy if indicated.

4 Dacryocystectomy, if indicated. If the nasal mucosa is unhealthy this may prove to be the better procedure.

Note. Dacryocystorrhinostomy and Dacryocystectomy should be done by a surgeon knowledgeable in these procedures.

\section{Examination of the eye in leprosy}

When examining the eyes of a person with leprosy, it is important to remember that he may have other conditions of the eyes not necessarily associated with leprosy; e.g. trachoma, cataracts, glaucoma, conjunctivitis, vitamin and nutritional deficiencies, significant refractive errors, etc. Just because leprosy is present, do not assume every abnormality is due to leprosy.

The following is a quick, simple, step-by-step eye examination of a patient with leprosy. If done properly, the vast majority of eye problems-both due to leprosy and other causes-should be diagnosed.

1 Observe patients for spontaneous blink (5th nerve). Is it frequent enough and symmetrically complete?

2 Ask the patient to close his eye 'as in sleeping' and with maximal effort ( 7 th nerve).

3 Examine the conjunctiva and cornea with a penlight (external diseases).

4 Check pupillary reaction with the penlight at the same time (iris atrophy, posterior synechiae due to uveitis).

5 Palpate the eye through closed eyelids to check for tenderness (scleritis, uveitis).

Note. Steps 1-5 required a penlight as the only piece of equipment. In most cases, 25-30 seconds per patient is adequate time for these steps.

The following two steps are more involved and should be done if possible:

6 Record the vision. With minimal training, an assistant can test the vision for the clinician to save time. Vision chart is required.

7 Intraocular pressure. This can be tested by a well-trained assistant. Requires a tonometer and a place for the patient to lie down. Clean the tonometer between patients. (If alcohol is used be sure to wipe the tonometer dry before using it again as alcohol can cause a painful keratitis.)

\section{Conclusion}

Ocular leprosy is a complicated medical condition; however, if the practitioner will use the information outlined in this paper and examine the eyes of many leprosy patients, he will find that the diagnosis and proper management of ocular leprosy is logical and attainable. 